Rhode Island College

Digital Commons @ RIC

May 2017

\title{
Nurses' Knowledge of Hypoglycemia and Treatment in the Adult Hospitalized Patient with Diabetes: A Quality Improvement Project
}

Brittney O'Neale

Rhode Island College

Follow this and additional works at: https://digitalcommons.ric.edu/etd

Part of the Nursing Commons

\section{Recommended Citation}

O'Neale, Brittney, "Nurses' Knowledge of Hypoglycemia and Treatment in the Adult Hospitalized Patient with Diabetes: A Quality Improvement Project" (2017). Master's Theses, Dissertations, Graduate Research and Major Papers Overview. 179.

https://digitalcommons.ric.edu/etd/179

This Major Paper is brought to you for free and open access by the Master's Theses, Dissertations, Graduate Research and Major Papers at Digital Commons @ RIC. It has been accepted for inclusion in Master's Theses, Dissertations, Graduate Research and Major Papers Overview by an authorized administrator of Digital Commons @ RIC. For more information, please contact digitalcommons@ric.edu. 



\title{
NURSES' KNOWLEDGE OF HYPOGLYCEMIA AND TREATMENT IN THE ADULT HOSPITALIZED PATIENT WITH DIABETES: \\ A QUALITY IMPROVEMENT PROJECT
}

\author{
A Major Paper Presented \\ by \\ Brittney Anne O’Neale
}

\section{Approved:}

Committee Chairperson

Committee Members

(Date)

(Date)

(Date)

Director of Master's Program

(Date)

Dean, School of Nursing

(Date) 


\section{NURSES' KNOWLEDGE OF HYPOGLYCEMIA AND TREATMENT IN THE ADULT HOSPITALIZED PATIENT WITH DIABETES: \\ A QUALITY IMPROVEMENT PROJECT}

by

Brittney Anne O’Neale

A Major Paper Submitted in Partial Fulfillment

of the Requirements for the Degree of

Master of Science in Nursing

in

The School of Nursing

Rhode Island College

2017 


\begin{abstract}
There are currently more than 29 million people in the United States with diabetes. With increasing numbers of diabetic patients across the nation, it is imperative that health care professionals receive the knowledge and skills required to manage them in the acute care setting. Hypoglycemia is the most common side effect of diabetes treatment (Borzi et al., 2016) and is defined by the American Diabetes Association (ADA) as a condition that occurs when one's blood glucose is lower than normal, usually less than $70 \mathrm{mg} / \mathrm{dl}$. Nurses' knowledge of hypoglycemia and their adherence to hospital protocols are essential to achieving positive patient outcomes. Research demonstrates that nurses in the acute care setting are not receiving consistent formal training on the care of adult diabetic patients and sometimes fail treat the patient according to evidence based hospital protocols. The purpose of this quality improvement project was to increase nurses' knowledge of hypoglycemia and treatment in the adult hospitalized patient with diabetes. The project design was a pre-test, educational program and post-test on two medicalsurgical units in a small community hospital in Rhode Island. Seventeen nurses completed the pre-test component $(\mathrm{N}=17,22 \%)$ and eighteen nurses $(\mathrm{N}=18,23 \%)$ attended the educational program and completed the post-test. Pre-test scores ranged from 11 to 94 out of a possible 100, with a mean score of 59.8\%. Post-test scores ranged from 27 to 100, with a mean score of $76.5 \%$. The average of post-test scores increased by about $16.7 \%$. These findings suggest that providing hypoglycemia education can be successful in increasing nurses' knowledge of hypoglycemia treatment and management in the adult hospitalized patient with diabetes.
\end{abstract}




\section{Acknowledgements}

I would like to thank Patricia Calvert for being my first reader and for your patience, support and guidance throughout this process. I would like to thank Cynthia Padula for being my second reader and Janet Engvall for being my third reader. To the nurse managers and nursing staff of 4 East and 4 West at The Miriam Hospital, thank you or your time and participation in this project. I would like to also thank Carly SheildsPirri, the Advance Practice Manager on 4 East and 4 West. Thank you for all your unwavering support throughout this project.

I would like to thank my friends and family who have supported me throughout this program. To my wonderful boyfriend Alex, with your encouragement I began this journey to obtain my Master's Degree. Thank you for all your love, support and patience throughout my graduate school experience. I truly could not have done this without your unconditional love and support. 


\section{Table of Contents}

Background/Statement of the Problem......................................

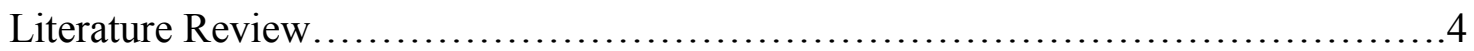

Theoretical Framework...................................................22

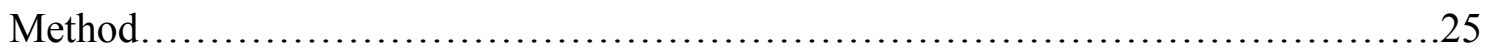

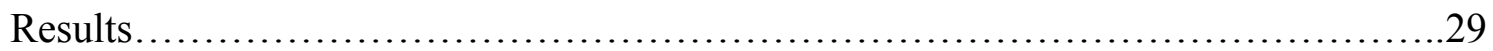

Summary and Conclusions................................................... 32

Recommendations and Implications for Advanced Nursing Practice..................35

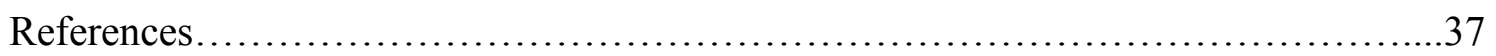

Appendices.................................................................... 41 
Nurses' Knowledge of Hypoglycemia and Treatment in the Adult Hospitalized Patient with Diabetes: A Quality Improvement Project

\section{Background/Statement of the Problem}

According to the Centers for Disease Control (CDC), there are 29.1 million people in the United States who have diabetes. Out of those, 21 million people are diagnosed and 8.1 million are undiagnosed (2014). The total cost of diagnosed diabetes in the United States as of March 2013 is estimated to be \$245 billion (American Diabetes Association [ADA], 2013) and the cost of in-patient hospital care of diabetes patients accounts for $43 \%$ of that total (ADA).

The incidence of diabetes, predominantly type 2 , has been rising rapidly since 1990 (Rowley \& Bezold, 2012). It is anticipated to increase by 64\% between 2010 and 2025, affecting 53.1 million people and increasing health care costs by $72 \%$ to $\$ 514$ million a year (Rowley \& Bezold). In Rhode Island, approximately 108,000 people are diagnosed with diabetes, with an annual cost of care totaling \$1.1 billion (ADA, 2015).

Hypoglycemia is the most common side effect of diabetes treatment (Borzi et al., 2016). The ADA defines hypoglycemia as a condition that occurs when one's blood glucose is lower than normal, usually less than $70 \mathrm{mg} / \mathrm{dl}$ (2014). Signs and symptoms include hunger, nervousness, shakiness, perspiration, dizziness, sleepiness, and confusion and may lead to unconsciousness if left untreated (ADA). Episodes of hypoglycemia increase length of hospital stay and increase the rate of hospital mortality in diabetic patients. In a study by Borzi et al. (2016), the risk factors for hypoglycemia in type 2 diabetic patients hospitalized in internal medicine units (IMUs) in Italy was studied. There was a significantly longer length of stay in patients with hypoglycemia compared 
to those with no hypoglycemia $(12.7 \pm 10.9$ versus $9.6 \pm 6.5$ days $)$. In-hospital mortality rate was $8.8 \%$ for patients who experienced hypoglycemia compared to $4.8 \%$ in those without hypoglycemia (Borzi et al.).

The mortality rate of patients who experienced hypoglycemia was also studied by McCoy et al. (2012). The retrospective study was conducted to determine whether a patients' self-report of severe hypoglycemia was associated with increased mortality. Patients who reported severe hypoglycemia increased their risk of death at 5 years by 3.4fold compared with those who reported no/mild hypoglycemia (McCoy et al.).

Hypoglycemia is a serious condition in diabetic patients that can lead to negative patient outcomes.

Nurses' knowledge of hypoglycemia treatment in the acute care setting is crucial for the care and safety of diabetic patients. In a study by Chinnasamy, Mandal, Khan, Iqbal, and Patel (2011), an audit was performed in their local practice in regard to inpatient hypoglycemia management. A questionnaire was developed to identify the gaps in nursing knowledge of hypoglycemia management. One hundred responses focusing on hypoglycemia treatment and management were evaluated using the questionnaire. The results showed that only $51 \%(n=51)$ of nurses were formally trained to manage hypoglycemia, but only $28 \%(n=28)$ successfully recognized the symptoms of hypoglycemia (Chinnasamy et al.).

Protocols are in place in most hospitals to properly manage hypoglycemia. However, a number of studies demonstrate that nurses fail to follow such protocols. One study in New Zealand examined nurses' adherence to their hospital's hypoglycemia protocol. A total of 117 hypoglycemia episodes were included. The protocol 
recommended oral treatment to correct hypoglycemia was 9-15 grams of glucose, but only $40.4 \%(n=46)$ of hypoglycemia cases were treated with the initial glucose recommendation. The protocol-required repeat glucose test in 10-15 minutes was only performed in $30.7 \%(n=35)$ of the cases. Only $36.7 \%(\mathrm{~N}=42)$ of hypoglycemia episodes were corrected within 30 minutes of discovery. Prolonged and recurrent hypoglycemia also occurred, with $40 \%$ of the episodes lasting more than one hour and $72 \%$ had more than one episode during their hospital stay (Coats \& Marshall). Nurses' knowledge of treatment protocols and adherence to hypoglycemia protocols in the acute care setting is necessary to prevent adverse short and long-term consequences of hypoglycemia episodes.

The purpose of this quality improvement project was to increase nurses' knowledge of hypoglycemia and treatment.

Next, a review of the relevant literature will be discussed. 


\section{Literature Review}

A literature review was conducted using the following databases and websites: Nursing Reference Center Plus; CINAHL; PUBMED; CDC; and ADA. Keywords included diabetes mellitus, hypoglycemia in diabetes, negative effects of hypoglycemia, hypoglycemia risk factors, hypoglycemia causes, nurses' knowledge of hypoglycemia, hypoglycemia treatment and management and hypoglycemia protocols.

\section{Diabetes Mellitus}

According to the ADA, diabetes mellitus is a condition characterized by hyperglycemia in which the body cannot break down glucose for energy (2014a). Most food is broken down into glucose or sugar and used for energy. The pancreas is an organ that makes insulin to break down glucose to use for energy in the body. In diabetes the pancreas can no longer make insulin, or cannot properly use the insulin produced by the pancreas, which causes sugar to build up in the blood (CDC, 2014). Some of the common symptoms of diabetes include frequent urination, increased thirst, increased hunger, extreme fatigue, blurry vision, cuts/bruises that are slow to heal, weight loss, tingling and pain or numbness in the hands and feet (ADA, 2014b).

There are two types of diabetes: type 1 and type 2 . Type 1 diabetes is an autoimmune disorder characterized by the destruction of insulin-producing beta cells in the pancreas (Zaccardi, Webb, Yates, \& Davies, 2015). The destruction of beta cells in the pancreas eventually leads to total insulin deficiency. Autoimmune diabetes accounts for about $90 \%$ of overall type 1 cases and is caused by both genetic and environmental factors that trigger the destruction of beta cells. Non-immune, or idiopathic diabetes, is 
much less common and occurs secondary to other diseases with varying degrees of insulin deficiency in which no autoimmunity is present (Zaccardi et al.).

Type 2 diabetes occurs when the pancreas can no longer produce enough insulin to break down glucose or the body is unable to use insulin properly. Type 2 diabetes is much more common than type 1, affecting $95 \%$ of the total number of individuals with diabetes in the United States (ADA, 2014). The interactions between genetic and environmental factors contribute to the incidence of type 2 diabetes. The most common risk factors are age, obesity, hypertension, dyslipidemia, sedentary lifestyle and family history (McCance \& Huether, 2014).

Treatment regimens for both type 1 and type 2 diabetes are prescribed to achieve optimal glucose control without causing hypoglycemia (McCance \& Huether, 2014). Diet, exercise and self-monitoring of blood glucose are included in the management of both type 1 and type 2 diabetes. For those with type 1 diabetes, some form of insulin must be incorporated in their treatment regimen. Treatment and management of type 2 diabetes varies based on the severity of the disease. Some individuals are able to manage their hyperglycemia with diet and exercise alone; others may need medications including insulin for optimal management. The risk for treatment-related hypoglycemia should always be considered while managing and treating diabetic patients (McCance \& Huether).

\section{Hypoglycemia: Pathophysiology, Causes, Risk Factors and Symptoms}

Pathophysiology. Hypoglycemia is a condition in which plasma glucose concentrations fall below $70 \mathrm{mg} / \mathrm{dl}$ (Briscoe \& Davis, 2006). The human brain depends on a continuous supply of glucose because it is unable to store this primary source of 
energy, so it is often the first organ to be affected by hypoglycemia. When blood glucose levels are lowered, a sequence of responses occur including the release of neuroendocrine hormones (or anti-insulin hormones), the activation of the autonomic nervous system (ANS), and neurogenic and neuroglycopenic symptoms are produced to protect the brain (Briscoe \& Davis).

Normally, the body's response to hypoglycemia consists of suppression of insulin release and secretion of glucagon and pancreatic polypeptides from the pancreas and the release of epinephrine, norepinephrine, cortisol and growth hormone (Briscoe \& Davis, 2006). Glucagon and epinephrine are the primary hormones that protect the human body against hypoglycemia. Both glucagon and epinephrine act to increase endogenous glucose production through increasing hepatic glycogenolysis and gluconeogenesis. If hypoglycemia is prolonged, the sympathetic nervous system (SNS) is activated, which results in increased release of free fatty acids. The free fatty acids result in glucose sparing because tissues can then oxidize free fatty acids instead of glucose (Briscoe \& Davis).

Causes. In a study by Smith, Winterstein, Johns, Rosenberg, and Sauer (2005), the causes of inpatient hyperglycemia and hypoglycemia were examined. Hypoglycemia events occurring in adult hospitalized patients were analyzed. Inclusion criteria consisted of patients who were admitted for at least 24 hours, over the age of 18 years and admitted to general medical or surgical service. There were a total of 188 hypoglycemic events with 24 events meeting the inclusion criteria. Eighteen of the 24 hypoglycemic events were considered to be preventable and were included in the analysis of the causes. Four causes of hypoglycemia were identified: response to divergence in the course of care; 
overall disease management; performance deficits; and patient behavior. Hypoglycemia events were attributed to disease management when there was a lack of understanding of appropriate glucose management. Events due to performance deficits resulted from providers failing to provide the correct treatment, even when they knew the treatment that should have been provided. Events caused by patients' behavior were when the event was attributed to the patient's behavior. The most common cause of hypoglycemia was response to divergence in the hospital course of care. Patients with reduced caloric intake without adjustments made to their antihyperglycemic medications who were then transferred to another floor for a procedure or diagnostic test were most frequently linked to hypoglycemia events. Antihyperglycemic medications were not adjusted based on decreases in oral intake or time spent in hospital procedure areas. Hypoglycemia events were most often related to prescribing, monitoring and communication between health care providers (Smith et al.).

The incidence of hypoglycemia in the hospital setting occurs frequently and is often predictable and/or preventable. In a study by Raijkanna, Martin, Whittle, and Oyibo (2013), the causes of hypoglycemia were identified using a Hypoglycemic Events Reporting System (HERS). The HERS is an audit tool developed to review the management of hypoglycemic events in the hospital and perform root cause analyses. All registered nurses who provided hypoglycemia treatments were asked to document each event using the HERS audit tool. A hypoglycemic event was defined as blood glucose of less than 4mmol/L. There were a total of 79 hypoglycemic events reported on the HERS occurring in 56 patients. Seventeen events $(22 \%)$ occurred while patients were taking insulin, a sulphonyurea or a combination of both and had decreased caloric intake due to 
associated illness. Twenty-three (29\%) events were attributed to insulin prescribing. Seventy percent of events occurred during the night or in early morning hours, indicating that the basal dose of insulin given the night before was too high. Upon assessment by the diabetes team, it was determined that $45.8 \%$ of the hypoglycemia events in this study were preventable (Rajkanna et al.).

In a large observational study in Sweden, Eriksson et al. (2016) compared the risk of cardiovascular disease, mortality and severe hypoglycemia in patients with type 2 diabetes starting on a second-line, non-insulin antidiabetic drug (NIAD). All patients with type 2 diabetes taking Metformin in combination with either a sulphonylurea, such as Glipizide, or a DPP-4 inhibitor, such as Januvia, were included. A total of 52,760 patients were included in this study that were treated with either metformin and a DDP-4 inhibitor or metformin and a sulphonylurea. Treatment with metformin and a sulphonylurea in type 2 diabetics was associated with an $88-107 \%$ increase in severe hypoglycemia when compared to metformin and a DDP-4 inhibitor. Patients with Type 2 diabetes treated with metformin and a DDP-4 inhibitor as a second line treatment had far fewer incidences of severe hypoglycemia (Eriksson, et al., 2016). In this study, dual treatment with a sulphonylurea was shown to increase severe hypoglycemia in type 2 diabetic patients.

Risk Factors. A single-center, cross sectional questionnaire survey study was conducted by Akram, Pedersen-Bjergaar, Carstensen, Borch-Johnsen, and Thorsteinsson (2006) that included patients with insulin-treated Type 2 diabetes. Patients participating in this study completed a questionnaire about their incidence of hypoglycemia in the past, hypoglycemia awareness and socio-demographic factors. All insulin-treated, type 2 
diabetic patients who had a previously arranged appointment at an outpatient diabetes center were recruited for this study. Patients treated with sulphonylureas, those with endstage renal disease (on dialysis), malignant disease, pregnancy or an inability to complete the questionnaire were excluded. Out of the 617 patients that were asked to participate, 417 returned a completed questionnaire. Based on the results of the questionnaire, hypoglycemia unawareness was the most significant risk factor for any event of severe hypoglycemia. The risk of severe hypoglycemia was increased 3 -fold (2.66 odds ratio) in those with hypoglycemia unawareness compared to those reporting normal hypoglycemia awareness (Akram et al.).

A retrospective, population-based study conducted by Honkasalo, Elonheimo, and Sane (2011) studied the risk factors for severe hypoglycemia (SH) in patients treated with anti-diabetic drug therapy. Both type 1 and type 2 diabetic patients were included in this study. The episodes of SH and their risk factors were identified using ambulance registers, databases of local health care units and patient questionnaires. Severe hypoglycemia was defined as a condition in which the patient required assistance from another person to recover. Of the type 1 diabetic patients treated with insulin, $14.6 \%$ $(n=91)$ needed ambulance or emergency room care due to SH episodes. A total of $31 \%$ $(n=22)$ of type 1 and $12.3 \%$ of type 2 diabetic patients receiving insulin therapy reported at least one episode of SH. Of all insulin treated patients, $53(7.8 \%)$ reported three or more episodes of SH in a one-year time frame. Treatment with insulin therapy was an independent risk factor for $\mathrm{SH}$ episodes in both type 1 and type 2 diabetic patients (Honkasalo et al.). 
In a study by Borzi et al., (2016) the risk factors for hypoglycemia in type 2 diabetic patients hospitalized on internal medicine units (IMUs) were studied. The purpose of this study was to describe the incidence of hypoglycemia, possible risk factors and the correlations with therapies in type 2 diabetic patients admitted to IMUs. A total of 3,176 diabetic patients were included in this study. Of that, $385(12.1 \%)$ experienced episodes of hypoglycemia during their hospitalization. A total of $44.2 \%(n=170)$ of older adult patients between the ages of 76 and 85 experienced episodes of hypoglycemia. Of the patients with hypoglycemia, $41 \%(n=158)$ had some degree of cognitive impairment. Nephropathy occurred in $23.9 \%$ (n=92) of patients with hypoglycemia. Hypoglycemia events were higher in patients treated with a sliding scale insulin $(19.4 \% ; \mathrm{n}=75)$ than those treated with basal bolus $(11.4 \%$; $n=44)$. There was a significantly longer length of stay in patients with hypoglycemia (12.7 days) compared to those with no hypoglycemia (9.6 days). Hypoglycemia was more prevalent in older adult patients, those with cognitive impairment, neuropathy, those on sliding scale insulin and those who had an increased length of hospital stay (Borzi et al.).

Symptoms. The symptoms of hypoglycemia can be categorized as neurogenic or neuroglycopenic (Briscoe \& Davis, 2006). Neurogenic or autonomic symptoms are triggered by an actively falling glucose, which causes the individual to realize they are having a hypoglycemic event. Neurogenic symptoms include shakiness, trembling, anxiety, nervousness, palpitations, sweating, clamminess, dry mouth, hunger pallor and pupil dilation. Neuroglycopenic symptoms are caused $b$ glucose deprivation in the brain. These symptoms include abnormal mentation, confusion, irritability, difficulty thinking, ataxia, paresthesias, headaches, stupor, seizures, coma and death (Briscoe \& Davis). 
Individual symptoms vary from person to person and it's important for individuals with diabetes to understand their personal hypoglycemic symptoms. It is important for diabetic patients to understand their body's reaction to hypoglycemia in order be able to effectively treat it and avoid severe hypoglycemia symptoms such as unconsciousness. The only definitive way to know if an individual is experiencing hypoglycemia is to test blood glucose (ADA, 2015).

\section{The Negative Effects of Hypoglycemia on Diabetic Patients}

Hypoglycemia is associated with severe adverse events among adult diabetic patients. A study conducted in Taiwan focused on the complications associated with hypoglycemia episodes in people with diabetes. Wu, Juang, \& Yeh (2011) conducted an exploratory and descriptive study collecting data through qualitative interviews to obtain participants' personal experiences with hypoglycemia. This study consisted of in-depth interviews with 17 participants, nine women and eight men. Inclusion criteria included: age 18 years or older; diagnosed with type 1 or type 2 diabetes for a minimum of 1 year; prior experience with hypoglycemia; willingness to openly share experiences and engage in dialogue; willingness to have audio recordings of their interviews and their signature on a consent form. There were four main themes that were discovered during the interviews. The first was an inability to control fluctuations in health. Ultimately, all the participants felt, in one way or another, that their diabetes and hypoglycemia episodes affected their daily routine. This was especially while at work and they reported often experiencing times when they were forced to ask for help from co-workers during a hypoglycemic event. The second theme referred to how hypoglycemia affects the participant's personal relationships such as family, friends, and colleagues. Stress to 
caregivers and family members was a common sub theme. Hypoglycemia episodes often resulted in patients being dependent on others for help and many felt they did not want to pose as a burden to others. Facing the disease alone was the third theme, which was associated with the difficult side effects and burdens that participants faced due to hypoglycemia. Participants explained that the behaviors associated with hypoglycemia events included seizures and behavioral changes that were embarrassing and cause them to feel isolated. Some participants feared that their symptoms of low blood sugar might be misconstrued as a drug addiction or a disability and feared the inability to maintain their job. Others identified lack of support by health care providers regarding their emotional responses to the disease. They felt as though health care providers were more concerned with their blood glucose levels and their ability to manage the disease independently, but rarely attempted to understand their personal experiences with the disease. The final theme was finding a balance between symptoms and how participants must understand their bodies in order to maintain safe blood sugar levels. Many participants expressed fears of both hypoglycemia and hyperglycemia and how the dilemma of obtaining a balance between the two is a constant battle. Having a good understanding of the patients' experiences with hypoglycemic episodes can help practitioners becoming more involved in promoting self-management. The authors identified the following key areas that health care providers should address related to diabetic patients with hypoglycemia episodes: concerns about personal relationships; facing the disease alone; dilemmas related to maintaining optimal blood glucose levels; and controlling fluctuations in health. The authors concluded that these key areas should 
be included in patient education and professional support for patients with diabetes $(\mathrm{Wu}$ et al.).

A retrospective study performed by McCoy et al. (2012) was conducted to determine whether patients self-report of severe hypoglycemia was associated with increased mortality. A total of 1,013 diabetic patients seen by a health care professional in a specialty diabetes clinic were included. The study was comprised of 216 patients with type 1 diabetes (21.3\%) and 797 patients with type 2 diabetes (78.7\%). Each participant was questioned about the frequency of hypoglycemia events during a preencounter interview and answers were recorded electronically in the diabetes electronic management system. Hypoglycemia events were characterized as mild or severe. Mild hypoglycemia was defined as symptoms such as dizziness, confusion and sweating that the patient was able to manage independently. Severe hypoglycemia was defined using the same symptoms, but required external assistance. Severe hypoglycemia was noted in 18 of the 140 patients (12.9\%) who were deceased compared to the 58 out of $873(6.6 \%)$ who were still alive. Five-year mortality was significantly higher among patients who reported severe hypoglycemia $(23.7 \%$; $\mathrm{n}=18)$ versus those who reported no/mild hypoglycemia $(13 \% ; n=122)$. Patients who reported severe hypoglycemia increased their risk of death at five years by almost 3.4-fold (OR 3.381 [95\% CI 1.546-7.388]) compared with those who reported no/mild hypoglycemia (OR 1.564 [95\% CI 0.9862.481]) (McCoy et al., 2012). These researchers concluded that severe hypoglycemia is a serious condition that can significantly increase mortality rates and should be included in the clinical assessment of every diabetes patient. 
A nationwide population-based study performed by Hsu et al. (2013) sought to identify the correlation between hypoglycemia and cardiovascular events and total mortality in patients with type 2 diabetes. Data were collected using the National Health Insurance program in Taiwan that insures $98 \%$ of the population. A cohort of 77,611 new diabetic patients was included in this study. There were a total of 1,844 hypoglycemia events. Of that total, 500 patients were identified as having severe hypoglycemia and 1,344 were identified as having mild hypoglycemia. In both mild and severe hypoglycemia events, hypoglycemia was most strongly associated with cardiovascular events including hypertension, atrial fibrillation, stroke and coronary heart disease (CHD). Hypertension was diagnosed in 63.6\% $(n=318)$ patients with severe hypoglycemia and $66.5 \%(\mathrm{n}=895)$ of patients with mild hypoglycemia. Atrial fibrillation was diagnosed in $1.2 \%(n=6)$ of patients with severe hypoglycemia and in $2 \%(n=27)$ of those with mild hypoglycemia. A diagnosis of heart disease was included in $13.2 \%$ (66) of patients with severe hypoglycemia and in $9.8 \%(n=132)$ of patients with mild hypoglycemia. Stroke was significantly higher in patients with severe hypoglycemia $(15 \% ; n=75)$ and somewhat higher in those patients with mild hypoglycemia $(11.1 \%$; $\mathrm{n}=150$ ). The findings indicated that hypoglycemia events, whether mild or severe, are strongly associated with subsequent cardiovascular events in type 2 diabetic patients. Hsu et al. also found that the hospitalization rate was significantly higher in patients with hypoglycemia during the same study period. Hypoglycemia patients had a 3.4-fold higher risk of being hospitalized with a hospitalization rate of $73.1 \%$. For patients with mild hypoglycemia, the first year hospitalization rate was $53.1 \%$ and for those with 
severe hypoglycemia the rate was $63.4 \%$. In addition, a total of $76.5 \%$ of hospitalizations occurred during the first year after the onset of hypoglycemia (Hsu et al.).

A cross-sectional, observational research study was conducted by Shi et al. (2014) combining patient-reported data collected through mail surveys as well as enrollment information from adult commercial health plan enrollees with type 2 diabetes. The purpose of this study was to test whether the fear of hypoglycemia in type 2 diabetic patients was associated with poorer health-related quality of life (HRQOL). Medical data, pharmacy data and enrollment information were collected from the Life Sciences Research Database, a large United States health plan. Participants included adult enrollees diagnosed with type 2 diabetes who were on $\geq 2$ oral antidiabetic medications (OAD). Health-related quality of life was evaluated using the EuroQol (EQ)-5D, a standardized measure that rates five dimensions of health including mobility, self-care, typical activities, pain and depression/anxiety across three levels (no problems, some problems, and extreme problems). The other tool used was a 12-item Short Form Health Survey Mental Component Summary (SF-12 MCS) and Physical Component Summary (SF-12 PCS). Scores range from 0 to 100; the higher the score, the better quality of life in physical and mental components. Fear of hypoglycemia was assessed using the Hypoglycemia Fear Survey (HFS), which assessed 15 behaviors that patients engage in to avoid hypoglycemia as well as 18 items related to low blood sugar. A total of 3,999 patients were contacted by mail and 813 responded with a compete survey. Out of the 813 responses, $578(71.1 \%)$ reported at least one hypoglycemia symptom. Patients who reported symptoms of hypoglycemia $(n=235)$ were associated with worse EQ-5D, SF-12 MCS and SF-12 PCS scores and a significantly higher HFS scores $(\mathrm{P}<0.0001)$, 
indicating increased fear of hypoglycemia consistent with decreased HRQOL.

Researchers concluded that fear of hypoglycemia was indeed independently associated with poorer overall health status and decreased quality of life (Shi et al.).

\section{Nurses' Knowledge of Hypoglycemia, Treatment and Management}

McEuen, et al. (2010) developed an evidence-based protocol for managing hypoglycemia. This program development project took place at Veterans Affairs Medical Center, a tertiary care teaching center with Magnet status. Because there was no standardized approach to hypoglycemia management, it was determined by the Clinical Practice Committee (CPC) that an evidence based hypoglycemia protocol was needed for nurses to safely and effectively treat hypoglycemia across multiple clinical areas. The CPC was a nursing committee charged with overseeing all of the documents associated with nursing practice including policies, procedures and protocols. The Hypoglycemia Workshop was formed which included certified diabetes educators and professional in nursing, pharmacy, medicine and nutrition. This interdisciplinary group created an action plan to collect and review existing evidence including the ADA 2009 clinical practice recommendations. Their existing protocol was modified to include current research on treatment options including those who were able to swallow or have gastrointestinal access, those with IV access or had an order to take nothing by mouth, and those who didn't have IV access. The treatment options for patients who were able to swallow included a 15g simple carbohydrate such as 1 tube of glucose gel or $4 \mathrm{oz}$ of fruit juice. Treatment options for patients with NPO status included administration of $25 \mathrm{ml}$ dextrose $50 \%$ intravenously. For patients with no IV access, confusion or loss of consciousness, $1 \mathrm{mg}$ of glucagon was to be administered intra-muscularly or subcutaneously. Blood 
glucose recheck was to be completed 15 minutes after each treatment intervention. With help from the nursing staff, a customized documentation template for a hypoglycemia episode was created. It was decided that the revised protocol would be adopted across all care areas. Once the protocol was approved, the Hypoglycemia Workshop implemented the protocol by ensuring the food and medication necessary for treatment was readily available to staff and conducted 30-minute training sessions over a two week period. To measure the effectiveness of the protocol, they evaluated the rates of severe hypoglycemia (blood glucose $<40 \mathrm{mg} / \mathrm{dL}$ ) before and after implementation of the protocol. Data from two medical and two surgical units revealed that rates of severe hypoglycemia varied widely during the five months prior to the implementation of the protocol. In the five months after protocol implementation, severe hypoglycemia rates decreased to very low levels across all four units, indicating that the implementation of an evidence-based hypoglycemia protocol was a safe and effective strategy for the management of hypoglycemia episodes (McEuen et al.).

Chinnasamy, Mandal, Khan, Iqbal and Patel (2011) used a questionnaire to identify gaps in nurses' knowledge of hypoglycemia management after finding several deficits in an audit of diabetic patient records. The questionnaire was developed based on the hospital's current hypoglycemia management guidelines. A total of 100 responses were received ( 80 staff nurses and 20 charge nurses). The results showed that only $51 \%$ $(n=51)$ of the nurses who participated in the study were formally trained on the management of hypoglycemia but $77 \%(n=77)$ were aware of the guidelines. Only $58 \%$ $(n=58)$ of the nurses recognized that a blood glucose of $<4 \mathrm{mmol} / \mathrm{L}(70 \mathrm{mg} / \mathrm{dl})$ indicates hypoglycemia. Only $28 \%(n=28)$ of nurses were able to identify all of the symptoms of 
hypoglycemia on the questionnaire and $29 \%(\mathrm{n}=29)$ were able to identify at least three symptoms. These findings suggest that many patients with hypoglycemia may go untreated and develop potentially serious symptoms. With regard to treatment, $73 \%$ of nurses responded that they would use some form of rapid-acting carbohydrate but only $10 \%$ responded they would use a quick-acting carbohydrate followed by a carbohydrate that is digested slowly. Only 33\% ( $\mathrm{n}=33$ ) of nurses responded that they would recheck a blood sugar in 15 minutes, and 28\% ( $\mathrm{n}=28)$ were unsure when to recheck. The results of this study showed that there was a lack of knowledge of hypoglycemia treatment and management. The authors concluded that providing mandatory education to nursing staff would help improve their knowledge and management of hypoglycemia.

In a pilot study by Engvall et al. (2014), a hypoglycemia protocol was developed based on the results of a research project in which nurses' knowledge of diabetes was examined. The purpose of this study was to identify baseline diabetes knowledge of direct care nurses and evaluate the effectiveness of an educational module. The study design included a pretest, educational module intervention and a posttest. An email was sent to 625 nurses asking them to participate in the study. A total of $150(18 \%)$ competed the pre-test and only 16 nurses completed the educational module and the posttest. Of the 150 nurses who completed the pre-test, 113 (76.9\%) of the respondents selected "Drink 4 oz. of orange juice with 2 teaspoons of sugar.” Only 13 (10.7\%) correctly answered, "Drink 4 oz. of regular soda," indicating a lack of knowledge of hypoglycemia treatment. As a result of the findings, the researchers developed a hypoglycemia protocol to guide nurses in hypoglycemia treatment measures. Researchers concluded that the development of a hypoglycemia protocol for medical surgical patients would empower 
nurses to treat hypoglycemia events independently while providing consistent treatment regimens for optimal patient outcomes (Engvall et al.).

\section{Nurse Compliance with Hypoglycemia Protocols}

Coats and Marshall (2013) conducted a retrospective chart audit of inpatient treatment orders and progress notes to examine nursing adherence to a hypoglycemia protocol. Adult patients admitted to two medical floors and two surgical floors with type 1 or type 2 diabetes who experienced an episode on inpatient hypoglycemia were included. Thirty-two cases met the inclusion criteria for the audit with a total of 117 hypoglycemic episodes. The hospital protocol identified blood glucose of $<4 \mathrm{mmol} / \mathrm{L}$ $(70 \mathrm{mg} / \mathrm{dl})$ as the clinical indicator for treatment. The protocol stated that a complex carbohydrate, such as a biscuit or chocolate milk drink, should only be administered after the hypoglycemia has been corrected using 3 to 5 vita tabs or $9-15 \mathrm{gm}$ of glucose. Upon detection of hypoglycemia, the correct amount of glucose was only administered in 46 (40.4\%) episodes. In 15 (13\%) hypoglycemic episodes, a complex carbohydrate rather than glucose was the first treatment provided. In 5 (4.3\%) episodes, no treatment was documented. A blood glucose recheck in 10-15 minutes post treatment was only completed in $35(30.7 \%)$ episodes. For patients who continued to experience hypoglycemia after the first treatment, retreatment with glucose was correctly administered to $18(25 \%)$ of the 72 patients. A complex carbohydrate was held when hypoglycemia persisted in $44(38.6 \%)$ of the episodes and once hypoglycemia was corrected, a complex carbohydrate was correctly provided in 44 (38.6\%) of episodes (Coats \& Marshall, 2013). Overall, the results of this audit demonstrated nurse 
compliance with the hypoglycemia protocol to be suboptimal indicating that further education and strategies to increase nurses' understanding of the protocol are needed.

Harrison, Stalker, Henderson and Lyerla (2013) conducted a retrospective study to evaluate if the use a clinical decision support system (CDSS) would improve compliance to specific hypoglycemia management guidelines. A CDSS is a computerized program within an electronic health record to support nursing decisions based on specific documented information. A total of 585 hypoglycemic episodes were reviewed in three phases. The phases included three different samples of 150 or more medical records of patients with diabetes who had at least one incident of hypoglycemia. Each was reviewed for nurse compliance with hypoglycemia protocol implementation and documentation. The first review was completed six months prior to implementing the intervention; the second review was completed six months following implementation; and the last review was completed 7-12 months after implementation. In phase 1, 284 episodes were reviewed, in phase 2, 150 were reviewed and in phase 3,158 were reviewed. Protocol adherence increased from $4 \%$ to $13 \%$ and then from $13 \%$ to $25 \%$ throughout the three phases (Harrison et al.). This study demonstrated that the use of a CDSS that gave nurses the ability to quickly access hypoglycemia guidelines improved compliance with the guidelines and ultimately improved patient outcomes.

A pilot project conducted by Cheuk, Yen, Chau, \& Ang (2015) sought to improve hypoglycemia management by nurses in two clinics. This study incorporated the Joanna Briggs Institute Practical Application of Clinical Evidence System (PACES) criteria into their current management of patients with hypoglycemia. Practical Application of Clinical Evidence System is an online tool used by health care personnel to conduct 
audits efficiently in the health care setting. The nurses who participated in the study attended an evidence-based education program which included nursing documentation according to the PACES criteria, definition of risk assessment of hypoglycemia, selfmonitoring of blood glucose, pathophysiology of insulin excess and compromised glucose counter-regulation. A pre and post implementation audit was performed on 17 nurses' documentation of hypoglycemia management to evaluate the improvement of hypoglycemia management after implementing the PACES criteria. There was a significant improvement in the findings between the pre and post intervention audits. There was a $6 \%$ improvement in the nurses' documentation of episodes of hypoglycemia and treatment. There was a $94 \%$ improvement in a repeated glucose documented 10 minutes post treatment. There was also a $94 \%$ improvement in the documentation of the risk assessment plan to identify patients with high risk of hypoglycemia and a $77 \%$ increase in documented evidence of daily blood glucose monitoring for patients with diabetes being treated with insulin (Cheuk et al.). The use of the PACES criteria was shown to significantly improve nurse's management of hypoglycemia, which could potentially reduce future hypoglycemic events and improve patient outcomes. Next, the theoretical framework guiding this project will be discussed. 


\section{Theoretical Framework}

\section{Knowles' Adult Learning Theory}

The theoretical framework used for this quality improvement project is Malcom Knowles' Adult Learning Theory. In Knowles' original work, he described the three steps in the adult learning process as the desire to learn, being actively involved in learning and the feeling of satisfaction or accomplishment (1950). Adults must be ready and motivated to learn and education material must be meaningful and satisfying to the adult learner. Knowles believed that adults learn differently than children and developed his theory of adult learning based on the concept of andragogy, which refers to any type of adult learning. Although there are currently five concepts of adult learning, Knowles' original work only included these four: self-concept; experience; readiness to learn; and orientation to learning (Knowles, 1970).

Knowles' described self-concept as an individual transitioning from being a dependent child to a self-directed human being (1970). It is the transition of child learners, into independent, self-directed adults. Children are completely dependent on adults for their every need and as they grow as full time learners, they develop their own identity and slowly begin to make decisions by themselves. As individuals grow and experience new things, they develop self-concept and a desire to become more independent and self-directed to make their own decisions. Once an individual begins to identify themselves as an adult, their role as a full time learner changes into that of a producer, or one who completely self-directed and able to make their own decisions to manage their life (Knowles). 
Adults have various levels of experience from which they develop throughout their life. Children and adults have very different definitions of what experiences are. A child defines an experience as something external, something that has happened to them that has affected them in some way. They also identify themselves based on external factors such as who their parents are, what street they live on, or which school they attend. Unlike children, adults identify themselves using their unique individual life experiences and are likely to identify themselves with their occupation, where they have traveled or what their achievements have been. Knowles described the differences in experience between adults and children as having these three consequences for learning: adults have more to contribute to the learning of others and they themselves are valuable resources for learning; adults have a greater volume of experience to relate to new experiences; and adults have developed fixed thought patterns and tend to be less open minded (Knowles).

Adults require a readiness to learn in order to be successful in development and growth. Children grow and develop through physiological and mental maturation where as adults tend to grow through the development of social roles. As adults transition through the stages of adulthood, their developmental tasks change in order for them to advance from one stage of life to the next. In order for adults to be successful learners, education must be relatable to their current task at hand and include real life experiences (Knowles, 1970).

Children learn through an accumulation of knowledge that is built on year after year through grade school (Knowles, 1970). Children build on what they have learned and use it to prepare themselves for the next stage of life. Adults tend to have the 
perception that education must be provided to accommodate their current life situation. Adults prefer to be educated on things that benefit their personal, professional, or social needs (Knowles).

In Knowles' theory, some of the basic learning concepts are crucial for educating adult learners. Nurses are adult learners that require education to be meaningful and appropriate to their current practice. It's important to incorporate these concepts while educating nurses on hypoglycemia treatment and management in order for them to fully engage in learning.

Next, the methodology for this quality improvement project will be presented. 


\section{Method}

\section{Purpose}

The purpose of this quality improvement project was to increase nurses' knowledge of hypoglycemia and treatment of the hospitalized adult patient with diabetes. Design

The design of this quality improvement project was a pre-test, educational intervention, and post-test.

\section{Participants and Site}

The potential participants included 77 registered nurses working on two medical surgical units, 4 East and 4 West, at The Miriam Hospital, a 247-bed tertiary care hospital located in Providence, Rhode Island. The Miriam Hospital is a Lifespan affiliated institution with five consecutive Magnet hospital designations. Convenience sampling was used and nurses were asked to voluntarily participate in this quality improvement project.

\section{Background}

The advance practice managers at the hospital collected data on nurse compliance with the existing hypoglycemia protocol over a 12-month period. Chart audits conducted on all in-patient units revealed that the nursing staff were not consistently following the Lifespan hypoglycemia protocol for diabetic patients with hypoglycemic episodes. An on-line learning module, developed in response to chart audit findings, failed to improve adherence to the hypoglycemia protocol. The education for this quality improvement project emphasized the most commonly missed elements of the hypoglycemia protocol identified in chart audits. 


\section{Procedures}

Administrative approval was obtained from nursing leadership and the nurse managers from 4 East and 4 West. Quality assurance (QA) determination was requested and received from both Lifespan IRB and Rhode Island College IRB. All nurses on the specified medical surgical unit were asked to participate. An informational flyer (Appendix A) was displayed in both staff lounges for one week prior to the start of the project. The Clinical Managers and the Advance Practice Manager (APM) distributed the flyer at daily huddles. An informational letter (Appendix B) was posted in the nurses' lounge to explain that the purpose of this project was to increase nurses' knowledge of hypoglycemia and treatment of the hospitalized adult patient with diabetes using a pretest, educational intervention, and post-test design. The letter further explained that participation was voluntary and no personal identifiers would be asked of participants.

One week after the flyer was distributed, and the informational letter was posted, copies of a 10-question multiple-choice pre-test (Appendix C) were placed in a manila envelope and were made available in the nurses' break room on both units. The informational letter, explaining the project was attached to the manila folder.

Nurses who chose to participate placed the completed pre-test in a locked box that was located in both of the break rooms. The nurses had two weeks to complete and submit the pre-test prior to the start of the interventional education program. At the end of the two-weeks the pre-tests were collected from the two units and locked in the advanced practice manager's office and were no longer accessible to the nurses.

\section{Educational Session}


Educational sessions occurred during the following two weeks. See Appendix D for program objectives and content outline. The 10-15 minute, brief educational sessions were offered on each unit during breaks. A poster board with the same information was used as a visual teaching aid at each presentation. Content included identifying signs and symptoms of hypoglycemia, treatment options for both the conscious and unconscious patient per hospital protocol, and timely repeat blood glucose checks. The poster board was taken down after each presentation and the post-test (Appendix C) was distributed immediately after each educational session. A pocket card (Appendix E) with bullet points of the Lifespan hypoglycemia protocol was distributed to all participating nurses at educational sessions after completion of the post-test. Candy was provided in exchange for the completion of the post-test. Participants then placed the post-tests in a locked box, and the locked box was locked in the APM's office after each educational session. The educational sessions were completed at the end of the two weeks and the pre/posttests compared.

\section{Measurement}

Development of the pre/posttest was based on the Lifespan hypoglycemia protocol and reviewed by a diabetes content expert, the managers on 4 East and 4 West, and the APM for both units. The 10-question multiple-choice pre-test and post-test was used to assess nurses' knowledge of hypoglycemia and treatment in the acute care setting before and after the educational intervention.

\section{Data Analysis}


A descriptive statistical analysis was used to measure the effectiveness of this quality improvement project. All of the answers on the pre-test were analyzed and compared to the answers on the post-test.

Next, the results will be presented. 


\section{Results}

Seventeen nurses out of a possible seventy-seven $(\mathrm{N}=17 ; 22 \%)$ completed the pretest portion of the project; an eighteenth pre-test was incomplete and not included in the pre-test results. Eighteen nurses out of a possible seventy-seven $(\mathrm{N}=18,23 \%)$ attended the educational session and completed the post-test. The test questions used in the pre-test and post-test are listed in Table 1. Comparison of test scores from the pre-test and posttest are illustrated in both Table 2 and Table 3.

Table 1 illustrates the pre-test/post-test questions.

\section{Table 1:}

\section{Pre-test/Post-test Questions}

1. Treatment is required for a hypoglycemia event when a patient's blood glucose is: (Circle the best answer)

2. When treating a patient with hypoglycemia which of the following are appropriate treatments for the conscious patient? (Circle all that apply)

3. After treatment of any hypoglycemia event, a repeat BG test must be completed within_minutes. (Circle the best answer)

4. If a blood glucose is notification to the responsible practitioner must take place within one hour using critical test reporting procedures. (Circle the best answer)

5. If your patient is having a hypoglycemic event, continue treatment until blood glucose reaches: (Circle the best answer)

6. If there is $\geq 1$ hour until the next meal post hypoglycemia event, provide a small snack of choice including: (Circle all that apply)

7. When treating a patient with a blood glucose that is $>40$ and $\leq 70$, who is unable to swallow, NPO, or rapidly deteriorating, treat immediately with: (Circle the best answer)

8. When you have a patient with a hypoglycemic event your documentation should include: (Choose the best answer)

9. Your patient has a blood sugar of 68 with no symptoms of hypoglycemia, the proper treatment would be to:

10. Your patient is alert and oriented $x 3$, with a blood sugar of 50, you treat the blood sugar appropriately per-protocol, your next steps would be: 
Table 2:

Test Score Comparison Pre-Test/Post-Test

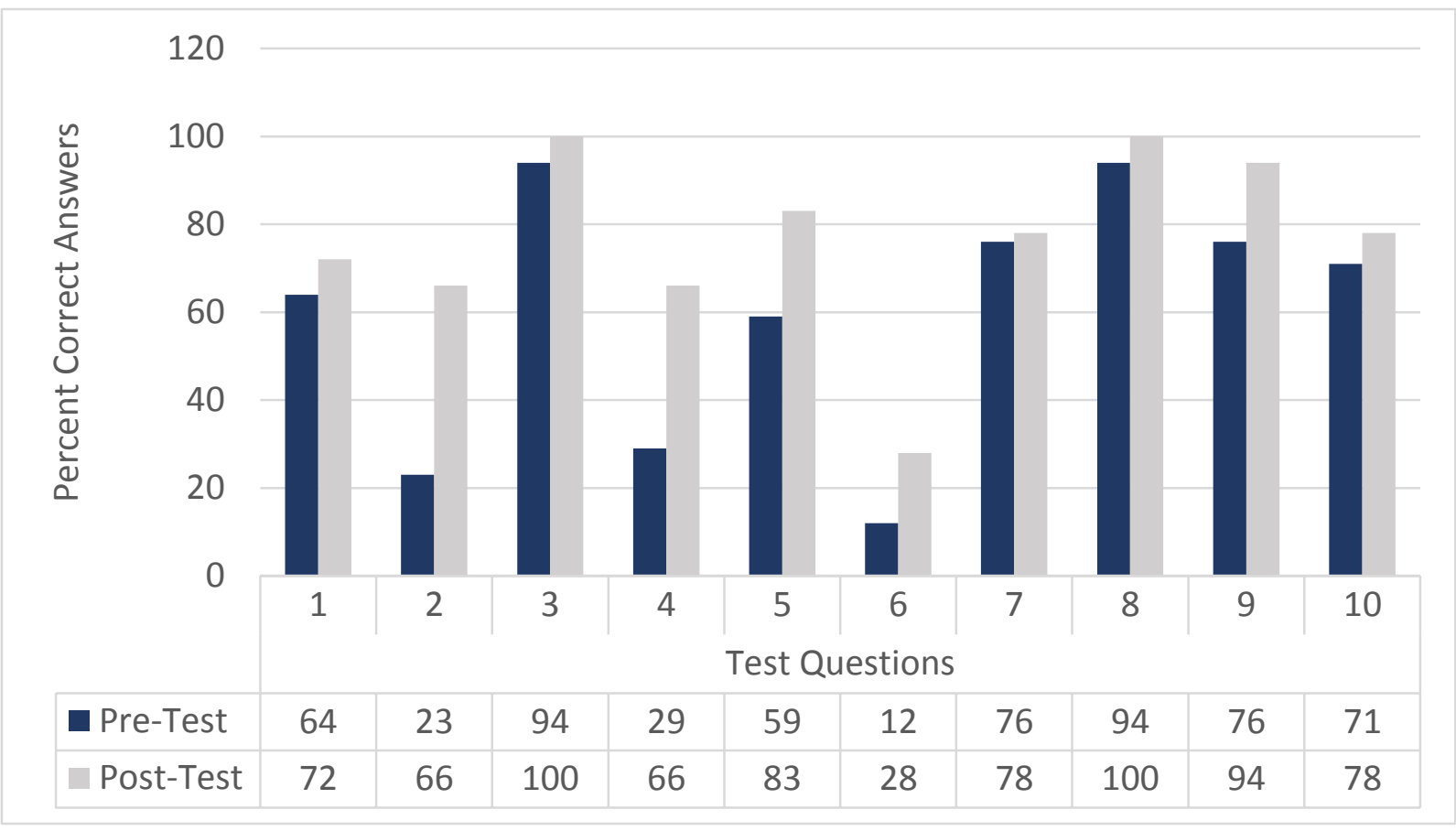

All post-test item scores improved when compared to the pre-test. Pre-test scores ranged from 11 to 94 out of a possible 100, with a mean score of 59.8\%. Post-test scores ranged from 27 to 100 , with a mean score of $76.5 \%$. The average of post-test scores increased by $16.7 \%$.

Questions number two and four demonstrated the most improvement between the pre-test and the post-test. Only 23\% (4/17) answered question number two correctly on the pre-test. After the educational sessions, $66 \%(12 / 18)$ were able to identify that the correct treatment for a conscious hypoglycemia patient is "four ounces of fruit juice and four ounces of regular soda" on the post-tests, an increase of $43 \%$. Only $29 \%(5 / 17)$ answered number four correctly and identified that a blood glucose level of $<55 \mathrm{mg} / \mathrm{dL}$ would require notification to the practitioner within one hour. After the educational sessions, $66 \%(12 / 18)$ were able to answer this question correctly, an increase of $37 \%$. 
Table 3 illustrates the percent correct for each of the questions on the pre-test and post-test.

Table 3:

Percentage Breakdown of Test Score Comparison Pre/Post-Test

\begin{tabular}{|c|c|c|}
\hline Question Number & Pre-Test (N=17) & Post-Test (N=18) \\
\hline 1 & $64 \%(11 / 17)$ & $72 \%(13 / 18)$ \\
\hline 2 & $23 \%(4 / 17)$ & $66 \%(12 / 18)$ \\
\hline 3 & $94 \%(16 / 17)$ & $100 \%(18 / 18)$ \\
\hline 4 & $29 \%(5 / 17)$ & $66 \%(12 / 18)$ \\
\hline 5 & $59 \%(10 / 17)$ & $83 \%(15 / 18)$ \\
\hline 6 & $12 \%(2 / 17)$ & $28 \%(5 / 18)$ \\
\hline 7 & $76 \%(13 / 17)$ & $78 \%(14 / 18)$ \\
\hline 8 & $94 \%(16 / 17)$ & $100 \%(18 / 18)$ \\
\hline 9 & $76 \%(13 / 17)$ & $94 \%(17 / 18)$ \\
\hline 10 & $71 \%(12 / 17)$ & $78 \%(14 / 18)$ \\
\hline
\end{tabular}

Pre-tests ranged from $12 \%$ to $94 \%$ correct answers on individual questions. The post-test ranged from $28 \%$ to $100 \%$ correct answers on individual questions. All of the questions demonstrated in increase in correct answers from the pre-test to the post-test. After the educational sessions, $100 \%$ of participants were able to answer two out of the ten questions correctly. Responses to question number six improved from pre-test to the post-test. However, scores remained low, with the percentage of correct answers increasing from $12 \%$ to $28 \%$. Most of the participants were still unable to identify the correct answer, "6 peanut butter crackers" and "four oz. milk with 3 soda crackers" on the post-test. An increase of correct answers was only $2 \%$ (from $76 \%$ to $78 \%$ ) on question number 7 which asked nurses to identify the correct treatment for hypoglycemia in a patient who is unable to swallow, NPO or rapidly deteriorating.

Next, the summary and conclusions will be discussed. 


\section{Summary and Conclusions}

The incidence of diabetes, predominantly type 2 , has been rising rapidly since 1990 and is anticipated to affect 53.1 million people by the year 2025 (Rowley \& Bezold, 2012). Hypoglycemia is the most common side effect of diabetes treatment (Borzi et al., 2016). According to the literature, episodes of hypoglycemia increase length of hospital stay and increase the rate of hospital mortality in diabetic patients. Research demonstrates that nurses in the acute care setting are not all receiving consistent formal training on the care of adult diabetic patients with diabetes and therefore may fail treat the patient according to evidence based hospital protocols.

Nurse compliance with the Lifespan hypoglycemia protocol for adult patients with diabetes was recognized as inconsistent through chart audits that were conducted on all in-patient units. A quality improvement project was developed using a pre-test, education intervention, post-test design to improve nurses' knowledge of the evidencebased hospital treatment guidelines for the treatment of hypoglycemia in the adult hospitalized patient with diabetes. The pre-tests were available to nursing staff over a two-week period to obtain the nurses' baseline knowledge. Over the next two weeks educational sessions were provided followed by the immediate distribution of the posttest. The pre-tests were then compared to the post-tests to assess whether or not the education improved nurses knowledge of the treatment of hypoglycemia in the adult hospitalized patient with diabetes.

A total of 17 nurses out of a possible 77 completed the pre-test. Eighteen out of a possible 77 nurses attended the educational sessions and completed the post-test. All of the post-test scores improved when compared to the pre-tests after the education was 
provided. The average post-test score increased by $16.7 \%$. All nurses who completed a post-test received a pocket reference card that included the information presented in the educational sessions.

Most nurses were able to correctly answer the questions on both the pre-tests and the post-tests, related to the time frame of repeat blood glucose checks after a hypoglycemia event and how to properly document the event. However, $100 \%$ of nurses answered these questions correctly after the educational intervention. There was a noticeable improvement on two questions. Question number two assessed the nurses' knowledge of the protocol driven treatment for a conscious patient with hypoglycemia " 4 oz. regular soda, 4 oz. fruit juice”, which improved from $23 \%$ on the pre-test to $66 \%$ on the post-test. Sixty-six percent of nurses knew that a blood sugar of $\leq 55 \mathrm{dL}$ required provider notification within one hour. Question number six assessed the nurses' knowledge of how to prevent a subsequent hypoglycemic event. Twelve percent of nurses were able to identify the correct answer as " 6 peanut butter crackers" and " $4 \mathrm{oz}$ milk with 3 soda crackers" on the pre-test. Despite the educational intervention, post-test percentage of correct answers remained low, only $28 \%$ of nurses were able to identify the correct answer. Proper treatment and timely provider notification are essential for positive patient outcomes when treating hypoglycemia.

Limitations were also evident. Due to the project design and anonymity of nurse responses on the pre-tests and post-tests, the number of nurses who completed all aspects of this project was undetermined. The overall percentage of staff participation in this project was low (22\%-23\%). Informational flyers and unit leadership encouragement were the only forms of advertisement for this project and were only posted for one week 
prior to the start of the project, which may have contributed to low pre-test participation. Providing education during breaks proved to be difficult at times. Hospital inpatient census was extremely high during the quality improvement project, which likely contributed to low post-test participation. Increased leadership support for nursing coverage was needed. Some participants who attended the educational sessions did not complete the post-test. Future replication of this quality improvement project would require more creative ways to recruit staff prior to the start of the project and provide more than one method to educate the staff.

Overall, the educational sessions proved to be successful for participating nurses, increasing knowledge of the treatment of hypoglycemia in adult hospitalized patients with diabetes. Future planning for an educational program related to this topic should increase the emphasis on how to prevent a subsequent hypoglycemia event when there is an hour or more before the next meal.

Next, recommendations and implications for advanced nursing practice will be discussed. 


\section{Recommendations and Implications for Advanced Nursing Practice}

The advanced practice nurse, specifically the Clinical Nurse Specialist (CNS) plays a major role in promoting and facilitating education of staff particularly related to evidence based practice and protocols. In this quality improvement project, the APRN student identified a hospital-wide need for hypoglycemia protocol education to improve nurses' knowledge of the treatment guidelines, documentation and prevention of hypoglycemia episodes. Adherence to the Lifespan hypoglycemia protocol is an important component of improved care and outcomes for hospitalized diabetic patients.

The APRN researches up-to-date, evidence based care and is in the position to play a key role in implementing a sustainable practice change in a health care. Advance practice nurses have expert clinical judgement and serve as leaders to advocate for safe patient care and improved outcomes. The APRN is critical in ensuring staff are able to understand current practices and protocols, and implement them successfully in the acute care setting. The APRN ensures policies are readily available to the staff nurse and reflect current evidence based practice.

Successful management of hypoglycemia in the adult hospitalized patient with diabetes requires collaborative interdisciplinary care. Advance practice nurses are in an ideal position to become leaders and advocate for improved patient outcomes through consistent education. Collaborating with other leaders and APRNs to reproduce the same education across the system could improve nurses' knowledge of hypoglycemia treatment and management.

The APRN has a broad perspective of the organization and is skilled in systems management, nursing education and patient care and plays a key role in the education of nursing staff on both the unit and systems level. The APRN is uniquely positioned to 
assess learning needs and has the ability to educate staff using various different methods in technology. Health care professionals utilize online educational modules to provide standardizing education for staff. Replication of the educational program presented in this quality improvement project can improve nurses' knowledge of the Lifespan hypoglycemia protocol and educate hospital wide how to effectively manage hypoglycemia in the adult hospitalized patient with diabetes. The APRN is viewed as an expert on evidence based practice and has the ability to collaborate with other disciplines and content experts to create online modules to effectively educate staff across the healthcare system.

Advance practice nurses consistently work with a broad range of technology in the health care setting. They are often involved in the technology selection process, implementation strategies, systematic problem solving and ensure that new interventions are consistent with evidence based care. Advance practice nurses are also responsible for the ensuring staff are adequately educated on new technology especially with regard to changes to the documentation system. They have the influence to make suggestions to the electronic documentation system especially when changes promote best practice.

As evidenced through the literature review for this quality improvement project, various tools and educational strategies have been developed to improve nursing knowledge of hypoglycemia management and protocol compliance. Consistent education and an easily accessible protocol for staff may have a positive impact on nurse education and increase protocol compliance. Future research is needed to identify barriers to successful management of hypoglycemia in the adult hospitalized patient with diabetes. 


\section{References}

Akram, K., Pedersen-Bjergaard, U., Carstensen, B., Borch-Johnsen, K., \& Thorsteinsson, B. (2006). Frequency and risk factors of severe hypoglycemia in insulin-treated Type 2 diabetes: a cross-sectional survey. Diabetic Medicine, 23(7), 750-756.

American Diabetes Association. (2013). The Cost of Diabetes. Retrieved from http://www.diabetes.org/advocacy/news-events/cost-of-diabetes.html American Diabetes Association. (2014a). Diabetes Basics. Retrieved from http://www.diabetes.org/diabetes-basics/common-terms/\#mellitus

American Diabetes Association. (2014b). Hypoglycemia (Low Blood Glucose). Retrieved from http://www.diabetes.org/living-with-diabetes/treatment-and-care/bloodglucose-control/hypoglycemia-low-blood.html?referrer=https://www.google.com/

American Diabetes Association. (2015). The Burden of Diabetes in Rhode Island. Retrieved from http://main.diabetes.org/dorg/PDFs/Advocacy/burden-ofdiabetes/rhode-island.pdf

Borzì, V., Frasson, S., Gussoni, G., Di Lillo, M., Gerloni, R., Augello, G., \& ... Fontanella, A. (2016). Risk factors for hypoglycemia in patients with type 2 diabetes, hospitalized in internal medicine wards: Findings from the FADOIDIAMOND study. Diabetes Research \& Clinical Practice, 11524-30. doi:10.1016/j.diabres.2016.01.020

Briscoe, V., \& Davis, S. (2006). Hypoglycemia in type 1 and type 2 diabetes: physiology, pathophysiology, and management. Clinical Diabetes, 24(3), 115-121. 
Centers for Disease Control and Prevention. (2014). CDC Report: 2014 National Diabetes Statistics Report. Retrieved from http://www.cdc.gov/diabetes/data/statistics/2014statisticsreport.html Centers for Disease Control and Prevention. (2015). What is Diabetes? Retrieved from http://www.cdc.gov/diabetes/basics/diabetes.html

Chinnasamy, E., Mandal, A., Khan, S., Iqbal, F., \& Patel, N. (2011). Nurses' knowledge of inpatient hypoglycemia management. Journal of Diabetes Nursing, 15(8), $313-317$.

Cheuk Ying, C., Yen Ling LEE, M., Chau Chain, Y., \& Ang, E. (2015). An evidence based implementation project: The management of hypoglycemia in patients with type 2 diabetes in primary care. Singapore Nursing Journal, 42(1), 15-21.

Coats, A., \& Marshall, D. (2013). Inpatient hypoglycemia: A study of nursing management. Nursing Praxis in New Zealand, 29(2), 15-24.

Engvall, J. C., Padula, C., Krajewski, A., Rourke, J., McGillivray, C. G., Desroches, S., \& Anger, W. (2014). Empowering the development of a nurse-driven protocol. MEDSURG Nursing, 23(3), 149-154.

Eriksson, J.W., Bodegard, J., Nathanson, D., Thuresson, M., Nystrom, T., \& Norhammar, A. (2016). Sulphonylurea compared to DDP-4 inhibitors in combination with metformin carries increased risk of severe hypoglycemia, cardiovascular events, and all-cause mortality. Diabetes Research and Clinical Practice. 117, 39-47.

Harrison, R. L., Stalker, S. L., Henderson, R., \& Lyerla, F. (2013). Use of a clinical decision support system to improve hypoglycemia management. MEDSURG Nursing, 22(4), 250-263. 
Honkasalo, M. T., Elonheimo, O. M., \& Sane, T. (2011). Severe hypoglycemia in drugtreated diabetic patients needs attention: A population-based study. Scandinavian Journal of Primary Health Care, 29(3), 165-170.

doi:10.3109/02813432.2011.580090

Hsu, P., Sung, S., Cheng, H., Yeh, J., Liu, W., Chan, W., \& ... Chuang, S. (2013). Association of clinical symptomatic hypoglycemia with cardiovascular events and total mortality in type 2 diabetes: A nationwide population-based study. Diabetes Care, 36(4), 894-900. doi:10.2337/dc12-0916

Knowles, M. S. (1950). Informal Adult Education. New York: Association Press.

Knowles, M. S. (1970). The Modern Practice of Adult Education. New York: Association Press.

McCance, K. L., \& Huether, S. E. (2014). Pathophysiology: The biologic basis for disease in adults and children. ( $7^{\text {th }}$ Ed.). Maryland Heights, MO: Mosby Elsevier.

McCoy, R., Van Houten, H., Ziegenfuss, J., Shah, N., Wermers, R., \& Smith, S. (2012). Increased mortality of patients with diabetes reporting severe hypoglycemia. Diabetes Care, 35(9), 1897-1901. doi:10.2337/dc11-2054

McEuen, J., Gardner, K., Barnachea, D., Locke, C., Backhaus, B., \& Hughes, S. (2010). Cultivating quality. An evidence-based protocol for managing hypoglycemia: Seeing the need for change and making changes. American Journal of Nursing, 110(7), 40-45. doi:10.1097/01.NAJ.0000383933.45591 
Rajkanna, J., Martin, D., Whittle, L., \& Oyibo, S. (2013). Hypoglycemia in hospital: A re-audit and root cause analysis using a hypoglycemic events reporting system. Practical Diabetes, 30(8), 336-338. doi:10.1002/pdi.1807

Rowley, W. R., \& Bezold, C. (2012). Creating Public Awareness: State 2025 Diabetes Forecasts. Population Health Management, 15(4), 194-200. doi:10.1089/pop.2011.0053

Shi, L., Shao, H., Zhao, Y., \& Thomas, N. A. (2014). Is hypoglycemia fear independently associated with health-related quality of life? Health \& Quality Of Life Outcomes, 12(1), 167-167. doi:10.1186/s12955-014-0167-3

Smith, W., Winterstein, A., Johns, T., Rosenberg, E., \& Sauer, B. (2005). Causes of hyperglycemia and hypoglycemia in adult inpatients. American Journal of Health-System Pharmacy, 62(7), 714-719.

Wu, F., Juang, J., \& Yeh, M. C. (2011). The dilemma of diabetic patients living with hypoglycaemia. Journal Of Clinical Nursing, 20(15/16), 2277-2285. doi:10.1111/j.1365-2702.2011.03725.x

Zaccardi, F., Webb, D., Yates, T., \& Davies, M. (2015). Pathophysiology of type 1 and type 2 diabetes mellitus: A 90-year perspective. Postgrad Medical Journal, 1084(92), 63-69. 
Appendix A

\section{Hypoglycemia Treatment in Adult Diabetic Patients:}

\section{What Every Nurse Needs to Know}

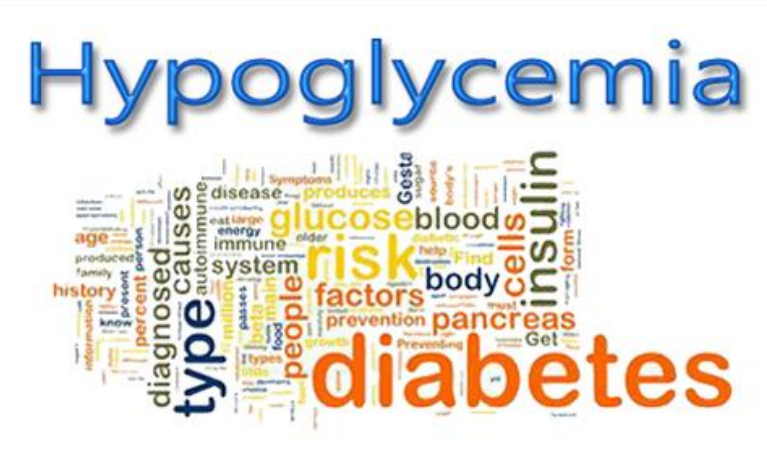

Educational Sessions- February 2017

4 East \& 4 West

The Miriam Hospital

For More Information Contact Brittney O'Neale at boneale_7232@email.ric.edu 


\section{Appendix B}

\section{INFORMATIONAL LETTER \\ Rhode Island College}

\section{NURSES’ KNOWLEDGE OF HYPOGLYCEMIA AND TREATMENT IN THE ADULT HOSPITALIZED PATIENT WITH DIABETES: A QUALITY IMPROVEMENT PROJECT}

Nursing Staff:

My name is Brittney O’Neale I am a Clinical Nurse Specialist student in the Masters of Science in Nursing program at Rhode Island College. I am currently conducting a quality improvement project on 4 East and 4 West. The purpose of this project is to improve nurses' knowledge of hypoglycemia and treatment in the adult hospitalized patient with diabetes. In order to complete this quality improvement project I am asking for nursing staff on 4 East and 4 West to participate. Participation includes completion an anonymous pre-test, attendance at a 10-15 minute educational session, and completion of an anonymous post-test. Pre-tests will be available to complete in each of the unit break rooms and placed in a locked box after completion. Educational sessions will be provided during lunch and dinner breaks and completion of the post-test will occur immediately after the educational sessions. Participation will remain voluntary and anonymous. If you have any questions please contact me at boneale_7232@email.ric.edu.

Thank you in advance for your participation, Brittney O’Neale, BSN, RN 


\section{Appendix C}

1. Treatment is required for a hypoglycemia event when a patient's blood glucose is: (Circle the best answer)
a. $\leq 60 \mathrm{mg} / \mathrm{dL}$
b. $<70 \mathrm{mg} / \mathrm{dL}$
c. $\leq 70 \mathrm{mg} / \mathrm{dL}$
d. $<80 \mathrm{mg} / \mathrm{dL}$

2. When treating a patient with hypoglycemia which of the following are appropriate treatments for the conscious patient? (Circle all that apply)
a. 4 oz. fruit juice
b. $4 \mathrm{oz}$. fruit juice with 2 packets of sugar
c. 4 oz. regular soda
d. 4 oz. milk with 3 soda crackers

3. After treatment of any hypoglycemia event, a repeat BG test must be completed within minutes. (Circle the best answer)
a. 15 minutes
b. 30 minutes
c. 45 minutes
d. 60 minutes

4. If a blood glucose is notification to the accountable LIP must take place within one hour using critical test reporting procedures. (Circle the best answer)
a. $<45 \mathrm{mg} / \mathrm{dL}$
b. $<50 \mathrm{mg} / \mathrm{dL}$
c. $<55 \mathrm{mg} / \mathrm{dL}$
d. $<60 \mathrm{mg} / \mathrm{dL}$

5. If your patient is having a hypoglycemic event, continue treatment until blood glucose reaches: (Circle the best answer)
a. $>100 \mathrm{mg} / \mathrm{dL}$
b. $\geq 90 \mathrm{mg} / \mathrm{dL}$
c. $>90 \mathrm{mg} / \mathrm{dL}$
d. $\geq 70 \mathrm{mg} / \mathrm{dL}$

6. If there is $\geq 1$ hour until the next meal post hypoglycemia event, provide a small snack of choice including: (Circle all that apply)

a. 1 whole meat sandwich

b. 6 peanut butter crackers

c. 4 oz milk with 3 soda crackers

d. $4 \mathrm{oz}$ fruit juice with 3 soda crackers

7. When treating a patient with a blood glucose that is $>40$ and $\leq 70$, who is unable to swallow, NPO, or rapidly deteriorating, treat immediately with: (Circle the best answer)

a. $50 \mathrm{~mL}$ of $50 \%$ Dextrose IV 
b. $25 \mathrm{~mL}$ of $50 \%$ Dextrose IV

c. $10 \mathrm{~mL}$ of $50 \%$ Dextrose IV

d. $5 \mathrm{~mL}$ of $50 \%$ Dextrose IV

8. Documentation of a hypoglycemic event should include: (Choose the best answer)

a. An event note with hypoglycemia treatment

b. An event note with hypoglycemia treatment and outcome

c. An event note including hypoglycemia treatment, the outcome, and provider notification

d. An event note with provider notification only

9. Your patient has a blood sugar of 68 with no symptoms of hypoglycemia, the proper treatment would be to:

a. Treat with $4 \mathrm{oz}$. of juice

b. Treat with milk and 3 soda crackers

c. No treatment is needed

d. Treat with $25 \mathrm{~mL}$ of $50 \%$ Dextrose IV

10. Your patient is alert and oriented $\mathrm{x} 3$, with a blood sugar of 50 , you treat the blood sugar appropriately per-protocol, your next steps would be:

a. Recheck the blood sugar within 30 minutes

b. Recheck the blood sugar within 30 minutes and notify the physician within 30 minutes

c. Recheck the blood sugar within $\mathbf{3 0}$ minutes, and notify the physician within one hour

d. Recheck the blood sugar within one hour, and notify the physician within one hour 


\section{Appendix D}

\section{OBJECTIVES}

- Describe the common signs and symptoms of hypoglycemia

- Describe treatment options for the conscious and unconscious patient per protocol

- Describe appropriate and timely blood glucose rechecks per protocol

- Describe how to prevent a hypoglycemia reoccurrence

- Describe how to document the hypoglycemia event in Life Chart per protocol

\section{CONTENT OUTLINE}

\section{HYPOGLYCEMIA SIGNS/SYMPTOMS}

\begin{tabular}{|c|c|c|}
\hline Mild & Moderate & Severe \\
\hline Trembling & Headache & Behavior changes \\
\hline Shaking & Fatigue & Confusion \\
\hline Cold, clammy skin & Weakness & Hallucinations \\
\hline Palpitations & Sudden vision changes & Amnesia \\
\hline Tachycardia & Mental dullness & Convulsions \\
\hline Diaphoresis & Lack of coordination & Coma \\
\hline Hunger & Unable to process information & \\
\hline Nausea & & \\
\hline
\end{tabular}

\section{HYPOGLYCEMIA TREATMENT}

- A blood glucose of $\leq 70 \mathrm{mg} / \mathrm{dL}$ is considered hypoglycemia and requires treatment

- There are two pathways to treating hypoglycemia:

○ Patients that are conscious, able to swallow, not NPO

○ Patients that are unable to swallow or NPO

\section{CONCIOUS PATIENT/ABLE TO SWALLOW}

- Treat with:

○ 4 oz. Fruit Juice

○ 4 oz. Regular Soda

- 4 oz. Apple Juice (renal insufficiency)

*Repeat glucose within 30 minutes

Continue to treat and recheck BG q 30 min until BG $\geq 90$

Contact LIP and document event under Significant Event. 


\section{UNABLE TO SWALLOW/NPO}

- $\mathrm{BG} \leq 70 \mathrm{mg} / \mathrm{dL}$ treat with:

○ $25 \mathrm{~mL}$ of $50 \%$ Dextrose

- $\mathrm{BG}<40 \mathrm{mg} / \mathrm{dL}$ treat with:

○ $50 \mathrm{~mL} 50 \%$ Dextrose

*Repeat glucose within 30 minutes

If $\mathrm{BG}$ remains $\leq 70$, repeat $25 \mathrm{~mL}$ Dextrose

Continue to treat and recheck BG q 30 min until BG $\geq 90$

*Contact LIP and document event under Significant Event.

\section{TO PREVENT REOCCURENCE}

- If there is $\geq 1$ hour until the next meal provide a small snack of choice:

- $1 / 2$ meat sandwich

- 6 peanut butter crackers

- 1 cup milk with 3 soda crackers

*NOTE: If BG is $<55$ notification to the responsible practitioner (MD/LIP) must take place within one hour using critical test reporting measures.

\section{EAST/ 4 WEST HYPOGLYCEMIA AUDIT RESULTS}

Timeline: January 2016- September 2016

- Follow-up blood glucose check within 30 minutes $=\mathbf{2 9} \%$

- Appropriate treatment documented $=\mathbf{5 8 \%}$

- Event note with LIP notification $=\mathbf{5 4 . 8 \%}$

- Follow up blood sugar within 30 minutes when second blood sugar is $<90=$ $11.7 \%$

- Total compliance with protocol $=\mathbf{1 3 \%}$ 


\section{Appendix E}

\begin{tabular}{|c|c|}
\hline$\frac{\text { Hypoglycemi }}{\text { Blood gluc }}$ & $\frac{\text { rotocol, Adult }}{\leq 70 \mathrm{mg} / \mathrm{dL}}$ \\
\hline Conscious patient, able to swallow & Cannot swallow, NPO, Unconscious \\
\hline $\begin{array}{l}\text { Treat with: } \\
4 \text { oz. Fruit Juice } \\
4 \text { oz. Regular Soda } \\
4 \text { oz. Apple Juice (renal insufficiency) } \\
\text { * Repeat glucose within } 30 \text { minutes } \\
\text { Continue to treat and recheck BG q } \\
30 \text { min until BG } \geq 90 \\
{ }^{*} \text { Contact LIP and document event. }\end{array}$ & $\begin{array}{l}\text { BG } \leq 70 \mathrm{mg} / \mathrm{dL} \text { treat with: } \\
25 \mathrm{~mL} \text { of } 50 \% \text { Dextrose } \\
\mathrm{BG}<40 \mathrm{mg} / \mathrm{dL} \text { treat with: } \\
50 \mathrm{~mL} 50 \% \text { Dextrose } \\
\text { * Repeat glucose within } 30 \text { minutes } \\
\text { If BG remains } \leq 70 \text {, repeat } 25 \mathrm{~mL} \\
\text { Dextrose } \\
\text { Continue to treat and recheck BG q } \\
30 \text { min until BG }>90 \\
\text { *Contact LIP and document event. }\end{array}$ \\
\hline
\end{tabular}

\section{To Prevent Reoccurrence:}

If there is $\geq 1$ hour until the next meal provide a small snack of choice:

$1 / 2$ meat sandwich

6 peanut butter crackers

1 cup milk with 3 soda crackers

*NOTE: If BG is $<55$ notification to the responsible practitioner (MD/LIP) must take place within one hour using critical test reporting measures. 